\title{
CDUNet: Cloud Detection UNet for Remote Sensing Imagery
}

\author{
Kai Hu ${ }^{1,2}$ (D), Dongsheng Zhang ${ }^{1}$ and Min Xia ${ }^{1,2, *(\mathbb{D})}$ \\ 1 Collaborative Innovation Center on Atmospheric Environment and Equipment Technology, Nanjing \\ University of Information Science and Technology, Nanjing 210044, China; 001600@nuist.edu.cn (K.H.); \\ 20201249164@nuist.edu.cn (D.Z.) \\ 2 Jiangsu Key Laboratory of Big Data Analysis Technology, Nanjing University of Information Science and \\ Technology, Nanjing 210044, China \\ * Correspondence: xiamin@nuist.edu.cn
}

Citation: Hu, K.; Zhang, D.; Xia, M. CDUNet: Cloud Detection UNet for Remote Sensing Imagery. Remote Sens. 2021, 13, 4533. https://doi.org/ $10.3390 /$ rs13224533

Academic Editors: Yang-Won Lee, Jungho Im, Jaeil Cho and Chu-Yong Chung

Received: 30 September 2021

Accepted: 10 November 2021

Published: 11 November 2021

Publisher's Note: MDPI stays neutral with regard to jurisdictional claims in published maps and institutional affiliations.

Copyright: (c) 2021 by the authors. Licensee MDPI, Basel, Switzerland. This article is an open access article distributed under the terms and conditions of the Creative Commons Attribution (CC BY) license (https:// creativecommons.org/licenses/by/ $4.0 /)$.

\begin{abstract}
Cloud detection is a key step in the preprocessing of optical satellite remote sensing images. In the existing literature, cloud detection methods are roughly divided into threshold methods and deep-learning methods. Most of the traditional threshold methods are based on the spectral characteristics of clouds, so it is easy to lose the spatial location information in the highreflection area, resulting in misclassification. Besides, due to the lack of generalization, the traditional deep-learning network also easily loses the details and spatial information if it is directly applied to cloud detection. In order to solve these problems, we propose a deep-learning model, Cloud Detection UNet (CDUNet), for cloud detection. The characteristics of the network are that it can refine the division boundary of the cloud layer and capture its spatial position information. In the proposed model, we introduced a High-frequency Feature Extractor (HFE) and a Multiscale Convolution (MSC) to refine the cloud boundary and predict fragmented clouds. Moreover, in order to improve the accuracy of thin cloud detection, the Spatial Prior Self-Attention (SPSA) mechanism was introduced to establish the cloud spatial position information. Additionally, a dual-attention mechanism is proposed to reduce the proportion of redundant information in the model and improve the overall performance of the model. The experimental results showed that our model can cope with complex cloud cover scenes and has excellent performance on cloud datasets and SPARCS datasets. Its segmentation accuracy is better than the existing methods, which is of great significance for cloud-detection-related work.
\end{abstract}

Keywords: cloud detection; cloud shadow; multiscale feature fusing; deep learning

\section{Introduction}

Pixel values indirectly reflect the spectral characteristics of ground objects, and different ground objects have different pixel values. Of course, excluding foreign objects with the same spectrum, an important symbol to identify different ground objects in remote sensing image interpretation is the difference of the pixel values of the images. The pixel value is also an important index reflecting the amount of information in an image.

The traditional threshold method [1-3] excessively relies on artificial calibration based on pixel values, that is the characteristics of clouds-high reflection and low temperatureand distinguishes clouds from ground objects by analyzing their Nearinfrared Spectrum (NIS). When it comes to complex situations, it is difficult to distinguish clouds using these manually calibrated characteristics. For example, Kegelmeyer [1] used simple cloud pixels as the threshold. Although this method is simple and feasible, it often leads to the omission or misjudgment of cloud detection. Zhu et al. [2] proposed an object-oriented Function of mask (Fmask) cloud detection method for remote sensing images captured by Landsat. This method uses cloud matching technology and a cloud height iterative algorithm for cloud detection. Experience has proven that the overall accuracy of cloud detection by the Fmask method is improved, but the detection rate of thin clouds is still not 
ideal. Candra et al. [3] added a Haze-Optimized Transformation (HOT) test and thermal band in the previous multitemporal cloud masking algorithm to improve the algorithm in the detection of haze, thin cirrus clouds, and thick clouds. They also improved the previous multitemporal cloud masking in the detection of cloud shadow by adding a blue band. Although their method is feasible and effective, the overall performance of cloud segmentation is limited.

With the rapid rise of deep learning, artificial neural networks are often used in cloud detection research because of their good universality. This kind of method can solve the problem of the temporal and spatial limitations of threshold methods to a great extent by training datasets. The advantage of using deep learning to detect clouds is that deep-learning models can automatically extract features from the images and complete end-to-end training [4,5]; this automatic feature extraction based on convolution networks provides higher accuracy for cloud and cloud shadow detection.

In recent years, many methods based on deep learning have been proposed to detect clouds, and multiscale feature fusion is often used in these methods. However, most existing methods fuse features through concatenation and elementwise summation, which are simple and can improve spatial information recovery. Besides, some cloud detection results are not accurate enough near the boundary of clouds. In view of the above problems, Wang et al. [6] proposed a cloud detection network, ABNet, which includes all-scale feature fusion modules and a boundary point prediction module. The all-scale feature fusion module can optimize the features and recover spatial information by integrating the features of all scales. The boundary point prediction module further remedies cloud boundary information by classifying the cloud boundary points separately. In addition, Xia et al. [7] proposed a global attention fusion residual network method to segment clouds and cloud shadow in satellite images. Meanwhile, the boundary refinement module was used to predict the boundary of clouds and shadows, so as to refine the boundary information.

Cloud and shadow segmentation is more challenging when only a limited number of spectral bands are available. To solve this problem, Mohajerani et al. (2019) [8] proposed an algorithm based on deep learning. The algorithm consists of a fully convolutional network [9], which is trained from multiple patches of Landsat 8 images. This network, known as cloud-net, is capable of capturing global and local cloud features in images using convolutional blocks.

Cloud and snow detection has significant remote sensing applications, while clouds and snow share similar low-level features due to their consistent color distributions and similar local texture patterns. Thus, accurately distinguishing clouds from snow at the pixel level from satellite images is always a challenging task with traditional approaches. To solve this shortcoming, Zhan et al. (2017) [10] proposed a deep-learning system for cloud and snow classification based on a pixel-level fully convolutional neural network using satellite images in three visible bands.

In addition, Dronner et al. [11] used the FCN architecture to detect clouds in multispectral remote sensing images. However, in the FCN framework, as it ignores the high-resolution feature image, the edge information is lost, so the segmentation results are poor in detail. Chai et al. [12] proposed a codec-based adaptive SegNet [13] for cloud and cloud shadow detection in Landsat images. However, SegNet has an insufficient grasp on the global semantic information and often makes misjudgments. Ronneberger et al. [14] proposed the U-shaped network (UNet), which can capture multiscale semantic information through repeated downsampling and upsampling of the deep network. However, due to the irregular geometry of clouds, it easily ignores the boundary information of fuzzy objects such as thin clouds:

- The location of clouds is often complex and diverse, and the distribution of clouds is often irregular and discontinuous. The traditional threshold method usually needs to use experience and manual calibration, so the prediction accuracy and universality are poor. Besides, the existing neural networks have defects in global information 
extraction, which easily lose the relative position information between clouds and shadows, so they easily cause category error detection;

- Cloud boundaries are often irregular geometric features. Therefore, we paid more attention to the prediction of cloud boundaries during model training. We hoped to complete the alignment directly in the end-to-end training process without the help of boundary postprocessing.

In view of the above findings, we propose a U-shaped structure model based on the deep-learning method, which uses ResNet [15] as the backbone network. ResNet joins the residual unit through a short-circuit mechanism. Because of its simple structure and excellent performance, it is often used as the backbone of segmented networks. The key to the performance of the U-shaped structure lies in the feature fusion stage. In the feature fusion stage, we used a High-frequency Feature Extractor (HFE) for the shallow input to extract the high-frequency information of the image, and at the same time, we used the Multiscale Convolution (MSC) to filter out the unnecessary high-frequency signal, so as to optimize the edge of the cloud. For the deep input, we integrated the Spatial Prior Self-Attention (SPSA) to obtain distance information between classes, so as to strengthen the control of global information and make the model have better spatial adaptability. In addition, we also introduced the Spatial Channel Attention Block (SCAB) to reduce the proportion of redundant information in the feature map and improve the efficiency of cloud detection. Finally, our model achieved a 93.52\% Mean Intersection over Union (MIoU) on the three cloud and cloud shadow classification datasets and showed the highest performance. In order to verify the generalization of the model, we also conducted experiments on the Star-Planet Activity Research CubeSat (SPARCS) dataset. The experimental results showed that our model is also ahead of the existing CNN model and can complete the cloud and cloud shadow segmentation tasks well. In summary, our research contributions are as follows:

- The traditional segmentation network will lose a portion of the detailed information in the downsampling stage. In order to refine the edge, we designed a high-frequency feature extractor to extract the detailed information of the image; convolution proved to be an effective feature extraction tool, and it also can be regarded as a special filter, so we designed a Multiscale Convolution module (MSC) to "filter" the image. While suppressing high-frequency noise, it extracts effective texture information, which can make the model predict more refined edges;

- The deep feature map contains lower-frequency signals, having stronger intraclass consistency, but weakening the interclass information. In order to re-establish interclass information, we designed a Spatial Prior Self-Attention block (SPSA) for the convolution neural network, which can better distinguish cloud, cloud shadow, and background information;

- A segmentation network based on an encoder-decoder will inevitably produce semantic dilution in the feature fusion stage. Other studies [14,16-18] simply combined high-level context information with Low-level spatial information to solve the above problems. This easily produces information redundancy, and consequently, the convolution kernel cannot receive effective feature information. In our method, we designed a parallel Channel Spatial Attention Block (SCAB) in the feature fusion stage, so that the model can quickly capture effective information and improve the prediction accuracy.

\section{Methodology}

Based on the above discussion, the semantic segmentation cloud detection model still needs improvement in the detail feature extraction and upsampling feature fusion stages. In this part, we first introduce the architecture of Cloud Detection UNet (CDUNet) and, then, focus on the feature fusion layer and its submodules: HFE, MSC, SPSA, and SCAB. 


\subsection{Network Architecture}

The accuracy of feature extraction directly affects the accuracy of the final classification $[19,20]$. Each layer of ResNet contains different levels of semantic information, which help us quickly extract multiscale information, and the residual structure of ResNet helps solve the problem of the gradient explosion and the vanishing gradient caused by the deepening of the network. Therefore, we used ResNet as the backbone network for remote sensing image segmentation. In addition, Lin et al. [21] proved that a $1 \times 1$ convolution can be used to change the number of channels, that is dimensionality reduction and increased information. The amount of calculation caused by the segmentation task is huge, and at the same time, to ensure the model's performance, we did not reduce the number of channels in the encoding stage, but used a $1 \times 1$ convolution in the decoder stage to reduce the number of channels to $1 / 2$ of the original, thereby reducing the overall parameters of the model. The overall structure is shown in Figure 1.

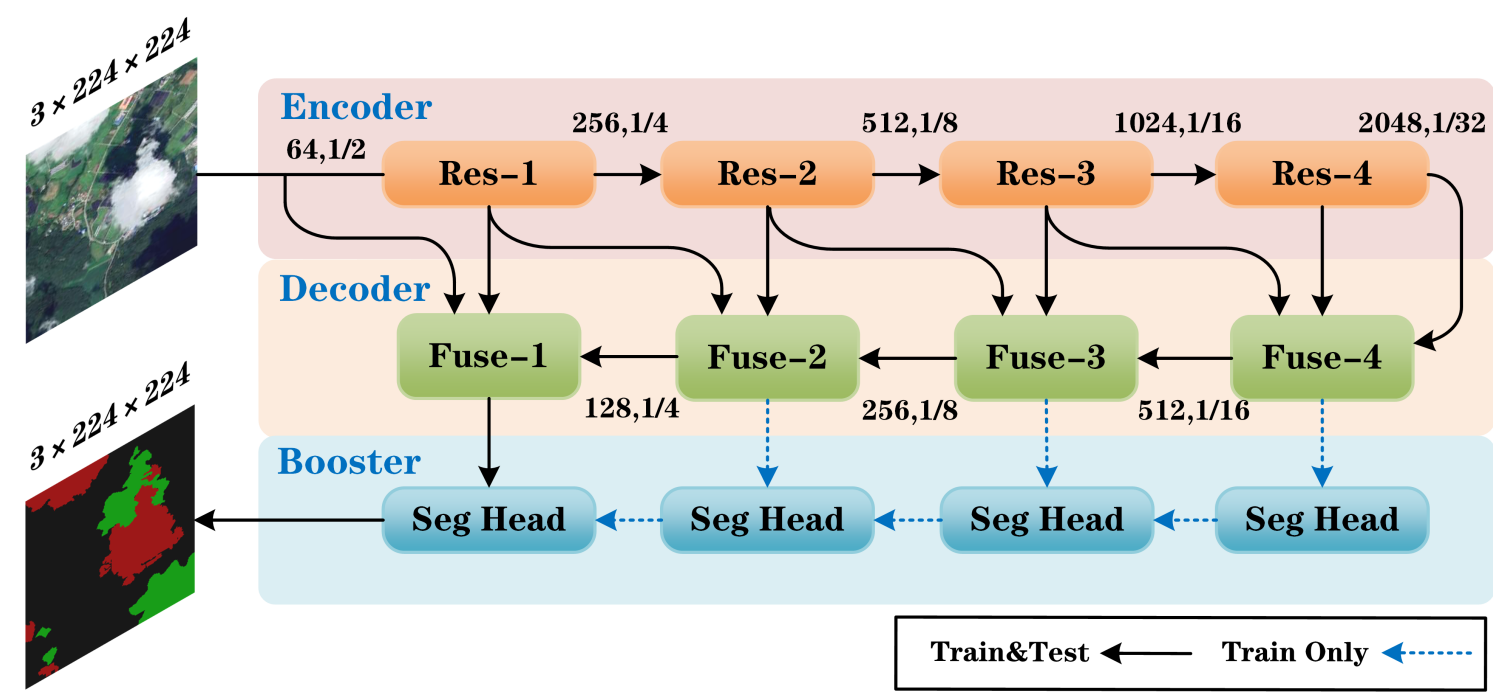

Figure 1. Framework of the proposed CDUNet. The framework is mainly composed of three parts: encoder, decoder, and booster. In the encoder stage, ResNet50 is responsible for extracting the semantic information; in the decoder stage, decoding information is extracted from the upsampling interaction layer; finally, the booster stage accelerates the segmentation efficiency and summarizes the information of each layer to complete the segmentation task.

In the decoder stage, the existing network cannot recover the lost details effectively. In order to solve this problem, we designed a feature fusion layer, which is also the most important part of CDUNet. As shown in Figure 2a,b, this layer is composed of the HFE, MSC, SPSA, and SCAB modules. As for the network function, the HFE module is mainly used to recover the details lost in the encoder stage; the MSC module is used to extract the multiscale information existing in the channel; the SPSA module is used to reconstruct the spatial location information from the deep network in the decoding stage; the SCAB module is used to improve the information redundancy in the feature map. From the aspect of the network structure, this layer is composed of two branches, one of which is a relatively high-resolution feature map generated from the shallow branch, and the other is a relatively low-resolution feature map with rich background information extracted through multiple downsampling operations. The two branches interact with the semantic information before entering the SCAB module. Each layer of the high-resolution and low-resolution representations receives information repeatedly, thus reducing the loss of semantic information and obtaining relatively rich feature representations. As shown in Figure 2c, Seg Head is a unique module of CDUNet in the training stage, which is composed of a convolution layer and a dropout layer. Its function is to speed up the convergence of the network and improve the prediction accuracy. 


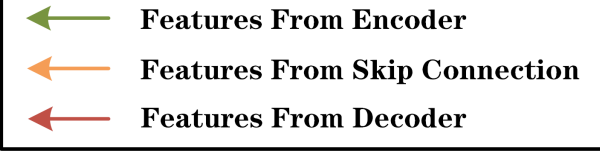

\section{Fuse-1}

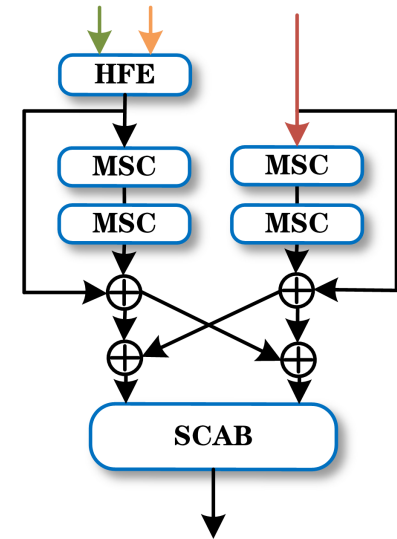

(a)

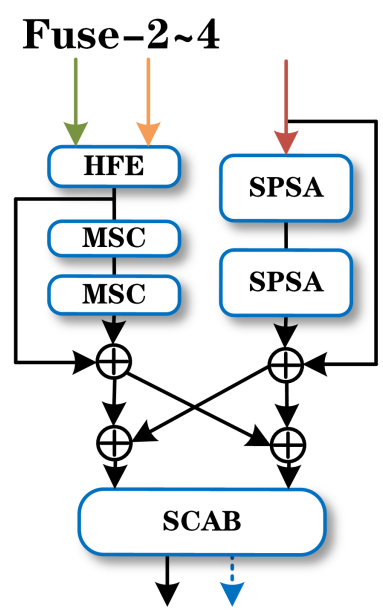

(b)

\section{Seg Head}

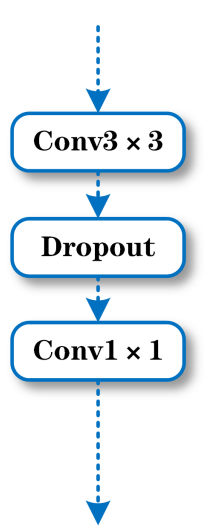

Figure 2. Proposed CDUNet structure: (a) Detail structure of Fuse Layer 1. (b) Detail structure of Fuse Layers 2-4. (c) Seg Head. $\oplus$ means elementwise add.

\subsection{High-Frequency Feature Extractor}

From the basic model of deep learning, LENet5, Reference [22] used the downsampling operation to reduce the information dimensionality, and the downsampling operation played a role in reducing the computational complexity and expanding the receptive field. At the same time, the low-pass filtering effect brought by the subsampling operation on the image is irreversible. In order to reproduce this part of the lost high-frequency information, we designed an HFE. In the encoder process, the classic network UNet [14] draws a semantic branch from each layer and passes it from the encoder to the decoder of the same number of layers through the concatenate operation, thereby introducing highresolution information, which can provide relatively fine features for segmentation, such as the edge of an object, etc. Our model continues this idea and introduces a high-frequency extractor to refine the high-resolution information brought by the shallow layer of the network. The method we used is to derive a branch from each layer of ResNet. This branch has not the undergone downsampling and convolution operations for the current layer, so it has rich semantic information. The details are shown in Figure 3. Its structure is simple and clear. Firstly, its input comes from two parts: one of them is from the shallow layer of the network, and the other is from the deep layer of the network. These two parts contain different characteristic information. Under ideal conditions, we believe that the former contains more information than the latter, or that the latter is a subset of the former. We make a convolution operation and upsampling operation on the deep feature map, enlarge it to the same size as the shallow feature map, and then subtract them. In this way, we obtain the relatively high-frequency feature map. Although the high-frequency signal is effective, it also has noise, so we set a learnable parameter to make the network adjust the proportion of the high-frequency signal independently. In this module, an addition operation is used to superimpose our high-frequency feature map on the original feature map to enhance the high frequency. 


\section{HFE}

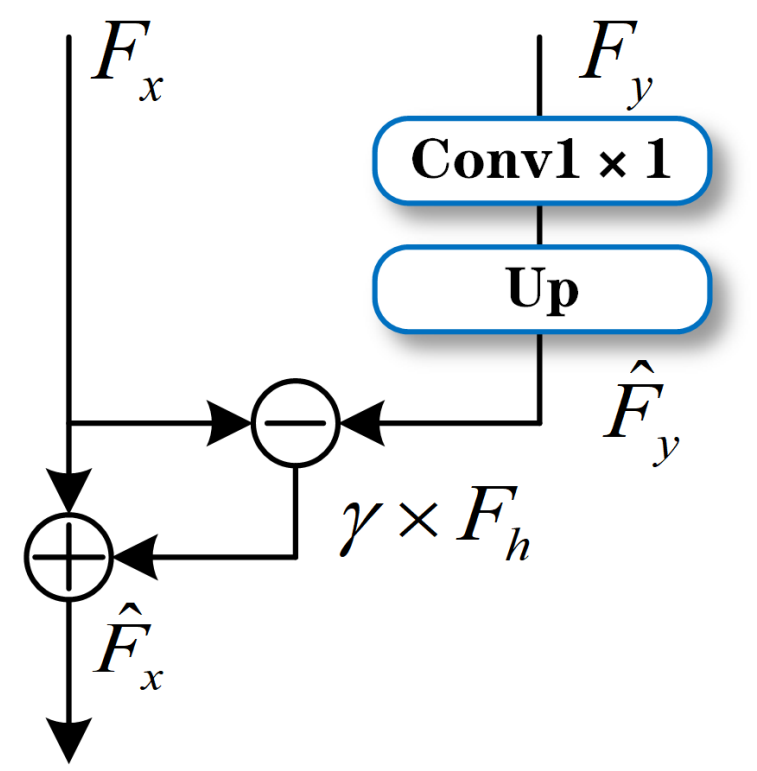

Figure 3. The structure of the high-frequency feature exacter, where $\oplus, \ominus$ represent elementwise add and subtract, respectively.

The design purpose of the module is to make up for the signal loss caused by the downsampling. The above steps can be expressed as follows:

$$
\begin{gathered}
\hat{F}_{y}=u p\left(\delta\left(F_{y}\right)\right), F_{y} \in \mathbb{R}^{h / 2 \times w / 2 \times c / 2}, \hat{F}_{y} \in \mathbb{R}^{h \times w \times c}, \\
F_{h}=F_{x}-\hat{F}_{y}, F_{x} \in \mathbb{R}^{h \times w \times c}, F_{h} \in \mathbb{R}^{h \times w \times c}, \\
\hat{F}_{x}=F_{x}+\gamma \times F_{h}, F_{h} \in \mathbb{R}^{H \times W \times C},
\end{gathered}
$$

where $\mathbb{R}$ represents the real number field, $h, w, c$ represent the height, width, and channel number of the feature graph, respectively, $F_{x}$ represents the shallow input, $F_{y}$ is the deep input, $\delta(\cdot)$ means the convolution operation is used to unify the number of channels, up $(\cdot)$ means the upsampling operation is used to unify the size of the feature map, $\hat{F}_{y}$ is the deep input after the upsampling and amplification, $F_{h}$ is the high-frequency characteristic map obtained by subtracting $F_{x}$ and $\hat{F}_{y}, \gamma$ represents the weight coefficient, and $\hat{F}_{x}$ is the final feature map after the high-frequency enhancement.

In order to verify the effectiveness of the module, we input three test images and used the heat map to represent the stage feature map. Figure $4 \mathrm{~b}$ represents the shallow input, while Figure 4c,d represents the deep input and the high-frequency characteristic graph obtained by the subtraction operation. Not surprisingly, we found that with the supplement of the shallow feature map, the final output feature map in Figure 4e has more abundant texture information. This shows that the HFE module effectively extracted the high-frequency features, and it was more accurate for the cloud and shadow location and the boundary prediction. 


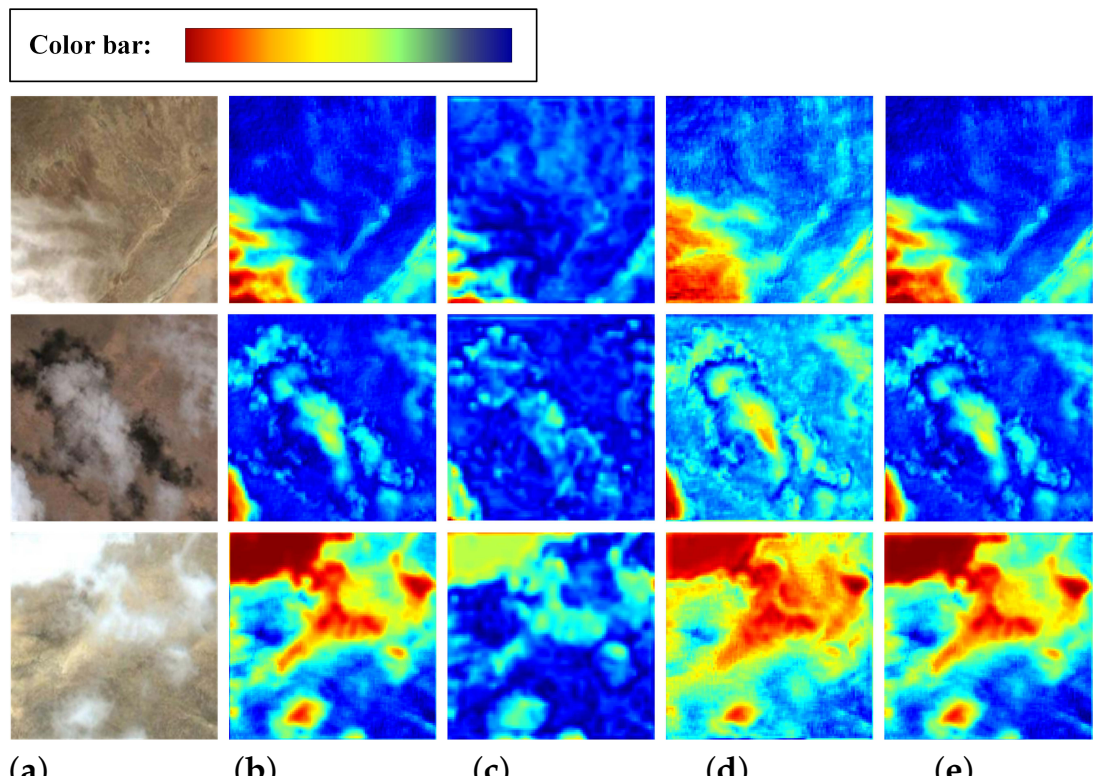

(a)

(b)

(c)

(d)

(e)

Figure 4. Heat map representation. (a) Test image; (b) shallow features; (c) deep features; (d) highfrequency features; (e) output features. The above images are from the cloud and cloud shadow datasets.

\subsection{Multiscale Convolution}

Although the HFE can extract the high-frequency information of the image, such as the boundary of the cloud layer and the sparse distribution of the point cloud, at the same time, noise is inevitably introduced, so we wanted to use a convolution layer to further filter the information. Representing features on multiple scales is very important for many visual tasks, and the majority of existing methods [23-25] represent multiscale features in a hierarchical way. We hoped to construct hierarchical residual class connections within a single residual block to represent multiscale features in a more fine-grained way and increase the receptive field of each network layer. In order to achieve the above functions, we designed an MSC module to filter the high-resolution feature map after the high-frequency extractor and further extract the multiscale features of the feature map.

As shown in Figure 5, except for the normal $3 \times 3$ convolution, we introduced a set of convolutions with kernel sizes of $5 \times 5$ and $7 \times 7$ to expand the receptive field and enrich the multiscale information. In order to reduce the number of parameters of the model, this design used the split method to perform the $5 \times 5$ and $7 \times 7$ convolutions in a parallel cascade, which has the same receptive field as the original convolution kernel. Due to the combination effect, many equivalent feature scales are generated. This structure can enhance the feature selection ability of the convolution kernel from the scale level. Specifically, the MSC first accepts the high-frequency fusion features from the HFE module. After a $1 \times 1$ convolution, we evenly divided the feature map $F_{i n}$ into $s$ feature map subsets, denoted by $x_{i}, i \in\{1,2, \cdots, s\}$. Each feature subset had the same size as the original feature map, but the number of channels was reduced to the original $\frac{1}{s}$. Other features except $x_{6}$ had a corresponding convolution layer, and the corresponding convolution operation is shown by $C_{i}(\cdot)$, so the output $y_{i}$ of each layer can be expressed by the following formula:

$$
\mathrm{y}_{\mathrm{i}}=\left\{\begin{array}{l}
C_{i}\left(x_{i}\right), i=1 \\
C_{i}\left(x_{i}+y_{i-1}\right), 1<i<s . \\
x_{i}, i=s
\end{array}\right.
$$




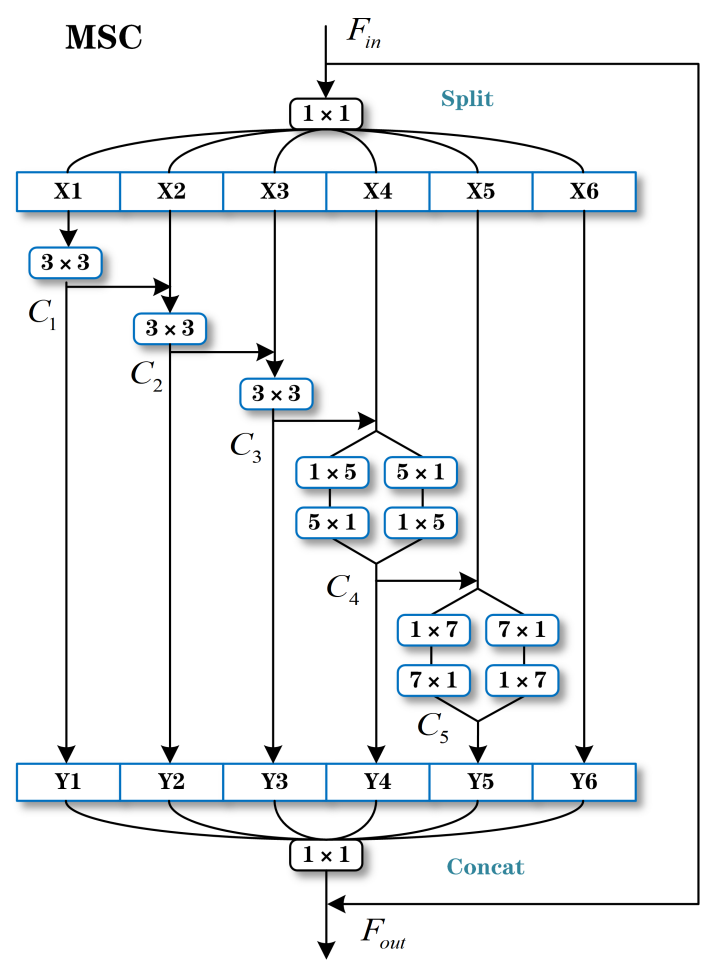

Figure 5. Multiscale convolution block.

After that, we merged the channels of each $\mathrm{y}_{i}$ and then through the $1 \times 1$ convolution, with the introduction of the residual structure. Finally, we obtained the desired feature map. In order to enhance the feature extraction ability, we reused two layers of the multiscaling in the feature fusion stage, and the details can be seen in Figure 2.

\subsection{Spatial Prior Self-Attention}

The work of Luo et al. [26] showed that the receptive field acted on by the convolution kernel is local and can only be correlated with the regions of different parts of the whole image after accumulating through many layers; that is because deep feature maps often contain more low-frequency signals, which have stronger intraclass consistency. When we consider the local feature aggregation of a pixel, we should pay attention to those pixels that have a greater impact on it. Therefore, we propose a spatial prior self-attention mechanism. This module uses the vector dot product to establish spatial location information between pixels, so that clouds and cloud shadows can be connected to the surrounding ground object information, so as to better distinguish the ground object information.

The nonlocal attention mechanism [27] proposed by Wang et al. uses vector dot multiplication to establish the spatial position relationship, but it takes up additional memory capacity, which is very unfriendly to the training process. DANet [28] attaches two types of attention modules to the backbone network to simulate the semantic interdependence in the space and channel dimensions, respectively; at the same time, DANet occupies much memory capacity. CCNet [29] obtains the pixel-level position information through the cross-path of each pixel, and then, through a circular operation, each pixel can capture the whole image dependency of all pixels. This cross-calculation method reduces the amount of calculation by losing a small amount of performance. As shown in Figure 6, we introduced an SPSA module to solve this problem. The difference from the above self-attention module is that we embedded two learnable vectors into the $\mathrm{K}$ vector, which is regarded as the horizontal and vertical spatial attention, which is equivalent to the introduction of a spatial prior. $P_{h}$ and $P_{w}$ represent the priors of the spatial position in the $H$ and $W$ dimensions respectively, which are a group of learnable vectors. They can update the gradients in the backpropagation, so as to optimize the pixels in the $H$ and $W$ dimensions and obtain the spatial attention map. The calculation formula is as follows: 


$$
\begin{gathered}
\hat{\theta}\left(x_{i}\right)=B N_{\gamma, \beta}\left(W_{\theta} x_{i}\right), \\
\hat{\phi}\left(x_{j}\right)=B N_{\gamma, \beta}\left(W_{\phi} x_{j}\right), \\
\hat{g}\left(x_{j}\right)=B N_{\gamma, \beta}\left(W_{g} x_{j}\right), \\
Z=\operatorname{softmax}\left((K+P)^{T} Q\right) V+X=\frac{1}{\sum_{\forall_{j}} e^{\left(\hat{\theta}\left(x_{i}\right)+p_{w}+p_{h}\right)^{T} \hat{\phi}\left(x_{j}\right)}} e^{\left(\hat{\theta}\left(x_{i}\right)+p_{w}+p_{h}\right)^{T} \hat{\phi}\left(x_{j}\right)} \hat{g}\left(x_{j}\right)+\sum_{\forall_{i}} x_{i},
\end{gathered}
$$

where $Q, K$, and $V$ represent the query, key, and value, respectively, $P$ is the prior of spatial position, $X$ represents the input feature map, $x_{i}$ represents the information of the current focus location, $x_{j}$ represents the global information, $\hat{\theta}(\cdot), \hat{\phi}(\cdot)$, and $\hat{g}(\cdot)$ respectively represent the $1 \times 1$ convolution and batch normalization operations. The final output is represented by $Z$. In addition, in order to reduce the amount of calculation, we adjusted the channel dimensions. We reduced the dimensions of the $k$ vector, the $q$ vector, and $P_{w}$ and $P_{h}$ in the channel dimension. Considering the amount of calculation, this module is only used on the low-resolution side of the feature fusion layer, and in order to improve the performance of the model in the feature fusion stage, we repeatedly used the SPSA module twice.

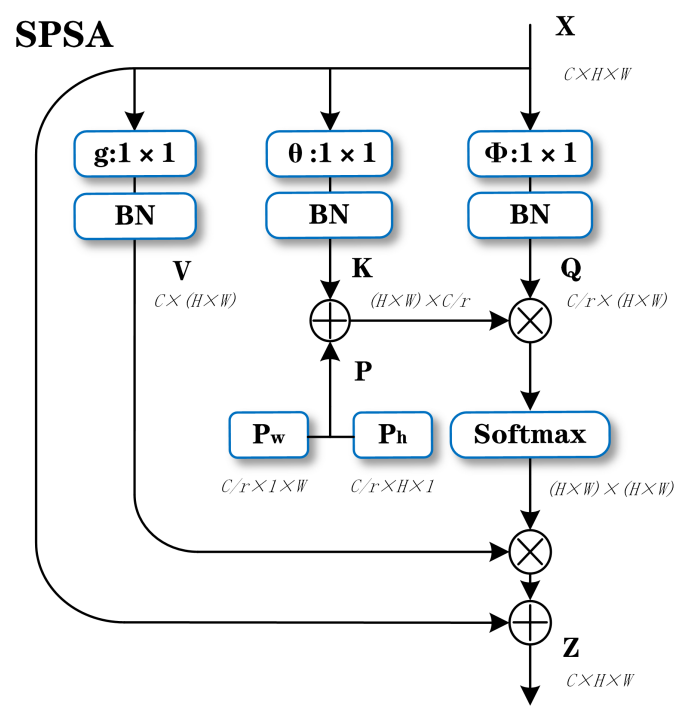

Figure 6. Spatial prior self-attention block. BN represents the Batch Normalization layer.

\subsection{Spatial Channel Attention Block}

In the feature fusion stage, the encoder-decoder-based segmentation network will inevitably produce semantic dilution, and most of these networks adopt simple feature graph addition or channel merge operations, which easily produce information redundancy, so that the convolution kernel cannot receive effective feature information. Inspired by $\mathrm{Li}$ et al. [30] and Woo et al. [31], in order to effectively integrate multilevel/multiscale feature maps, we designed a parallel spatial channel attention mechanism in the feature fusion stage, so as to enable the model to quickly capture effective information and improve the prediction accuracy.

As shown in Figure 7, we used the cross-branch relationship between the two input branch feature maps to generate spatial channel attention weights; that is, feature map $X \in \mathbb{R}^{h \times w \times c}$ is downsampled and added to feature map $Y \in \mathbb{R}^{h / 2 \times w / 2 \times c}$ to form attention map $U \in \mathbb{R}^{h \times w \times c}$. The maximum pooling and average pooling operations are used to generate the spatial attention map, and then, two layers of convolution are used to extract the spatial information. 


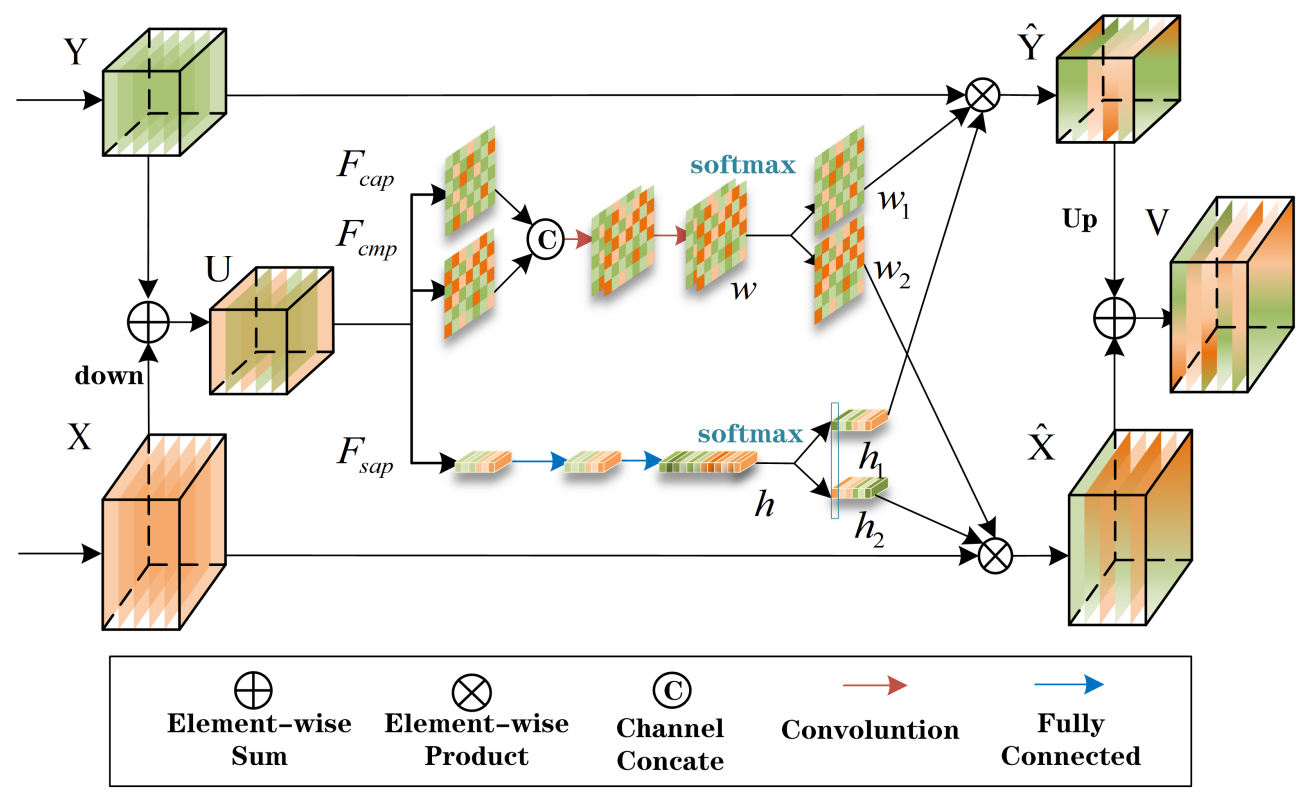

Figure 7. Spatial channel attention block.

The final spatial attention map $w \in \mathbb{R}^{h \times w \times 2}$ can be expressed by the following formula:

$$
\begin{gathered}
U=Y+\sum_{i \in h \times w} \frac{X_{i}}{s^{2}}, \\
\mathrm{w}=f^{1 \times 7}\left(f^{7 \times 1}\left(\left[F_{c a p}(U) ; F_{c m p}(U)\right]\right)\right),
\end{gathered}
$$

where $s$ represents the downsampling step size, which was set to two here; $F_{c a p}(\cdot)$ and $F_{\text {cap }}(\cdot)$ are the global average pooling and global maximum pooling for aggregating channel information; $f^{1 \times 7}$ and $f^{7 \times 1}$ represent the $1 \times 7$ and $7 \times 1$ convolution operations, respectively. Similarly, we used the average pooling to generate channel feature $e \in \mathbb{R}^{1 \times 1 \times c}$ in the spatial dimension of $U \in \mathbb{R}^{h \times w \times c}$ and, then, used two fully connected layers to extract the channel information, so that the channel attention map $h \in 1 \times 1 \times 2 c$ can be expressed by the following formula:

$$
h=f_{f c 1}\left(f_{f c 0}\left(F_{s a p}(U)\right)\right),
$$

$F_{\text {sap }}(\cdot)$ is the global average pooling for aggregating spatial information. $f_{f c 1}$ and $f_{f c 0}$ represent the full connection layer. Then, we used the softmax operation for the spatial attention map and channel attention map to obtain the spatial and channel attention weights, that is:

$$
\begin{aligned}
\left(w_{1} ; w_{2}\right) & =\sigma(w), \\
\left(h_{1} ; h_{2}\right) & =\sigma(h),
\end{aligned}
$$

where $\sigma(\cdot)$ is the softmax operator, $w_{1} \in \mathbb{R}^{h \times w}$ and $w_{2} \in \mathbb{R}^{h \times w}$ represent the spatial weights of $Y$ and $X$, respectively, and $h_{1} \in \mathbb{R}^{c}$ and $h_{2} \in \mathbb{R}^{c}$ represent the channel weights of $\mathrm{Y}$ and $\mathrm{X}$, respectively. The high and low resolution features $\hat{\mathrm{Y}} \in \mathbb{R}^{h \times w \times c}, \hat{\mathrm{X}} \in \mathbb{R}^{h / 2 \times w / 2 \times c}$ and the final fusion feature $\mathrm{V} \in \mathbb{R}^{h \times w \times c}$ can be calculated as follows:

$$
\begin{gathered}
\hat{Y}=w_{1} \otimes Y+h_{1} \otimes Y, \\
\hat{X}=w_{2} \otimes X+h_{2} \otimes X, \\
V=u p(\hat{Y})+\hat{X},
\end{gathered}
$$

where $\otimes \mathrm{a}$ is the matrix multiplication and $u p(\cdot)$ is the two-times upsampling. Finally, we obtained the feature map optimized by the spatial channel attention. 
As shown in Figure 8, we added the attention map to the original image to obtain the above images. From the graph, we can see that after adding the channel space attention model, our model will pay more attention to the effective information of the network prediction and make the prediction target clearer.

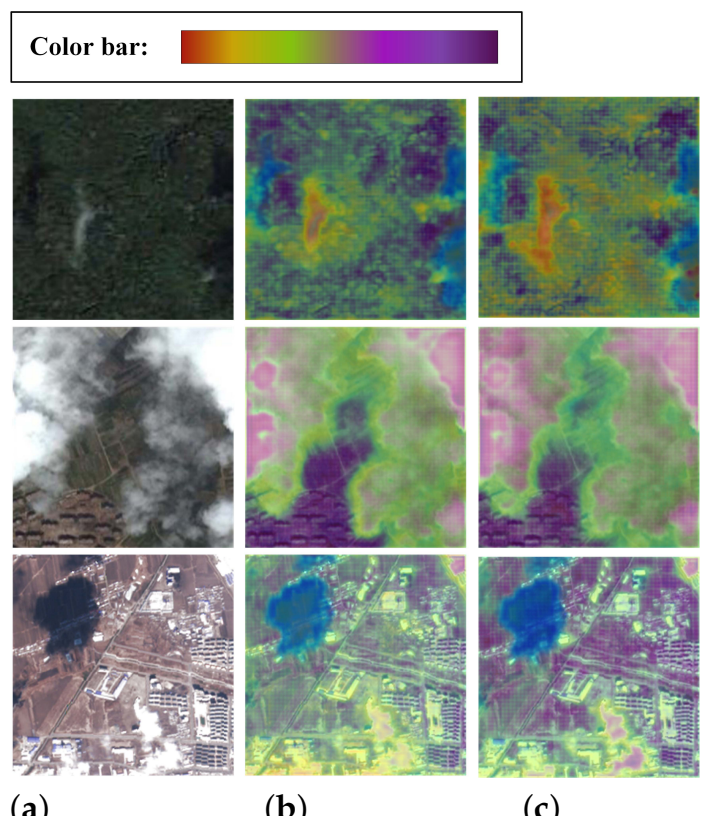

(a)

(b)

(c)

Figure 8. Heat map representation. (a) Test image; (b) model without the SCAB; (c) model with the $\mathrm{SCAB}$. The above images are from the cloud and cloud shadow datasets.

\section{Experiment}

\subsection{Dataset Introduction}

\subsubsection{Cloud and Cloud Shadow Dataset}

The cloud and cloud shadow datasets used in this paper were from Google Earth, which is a virtual Earth software developed by Google. It puts satellite photos, aerial photos, and the GIS on the 3D model of the Earth. The effective resolution of global geomorphologic images on Google Earth is at least $100 \mathrm{~m}$, usually $30 \mathrm{~m}$ (for example, Mainland Chinese), and the altitude of the view (Eyealt) is $15 \mathrm{~km}$. The dataset is composed of high-definition remote sensing images randomly collected by professional meteorological experts in Qinghai, the Yunnan Plateau, the Qinghai Plateau, the Qinghai Tibet Plateau, and the Yangtze River Delta. In order to better reflect the performance of the model, we selected several groups of high-resolution cloud images with different shooting angles and heights. Due to the limitation of the GPU video memory capacity, we cut the high-definition cloud remote sensing image with the original resolution of $4800 \times 2692$ to a size of $224 \times 224$. After screening, we obtained a total of 9217 images, among which 7374 were used as the training set and 1843 as the verification set. The ratio of the training set to the verification set in the dataset was 8/2. As shown in Figure 9, the high-resolution cloud and cloud shadow images derived from Google Earth are roughly divided into 5 types, which have different backgrounds, namely water area, woodland, farmland, city, and desert. The tags are manually tagged and come in three types: cloud (red), cloud shadow (green), and background (black). 


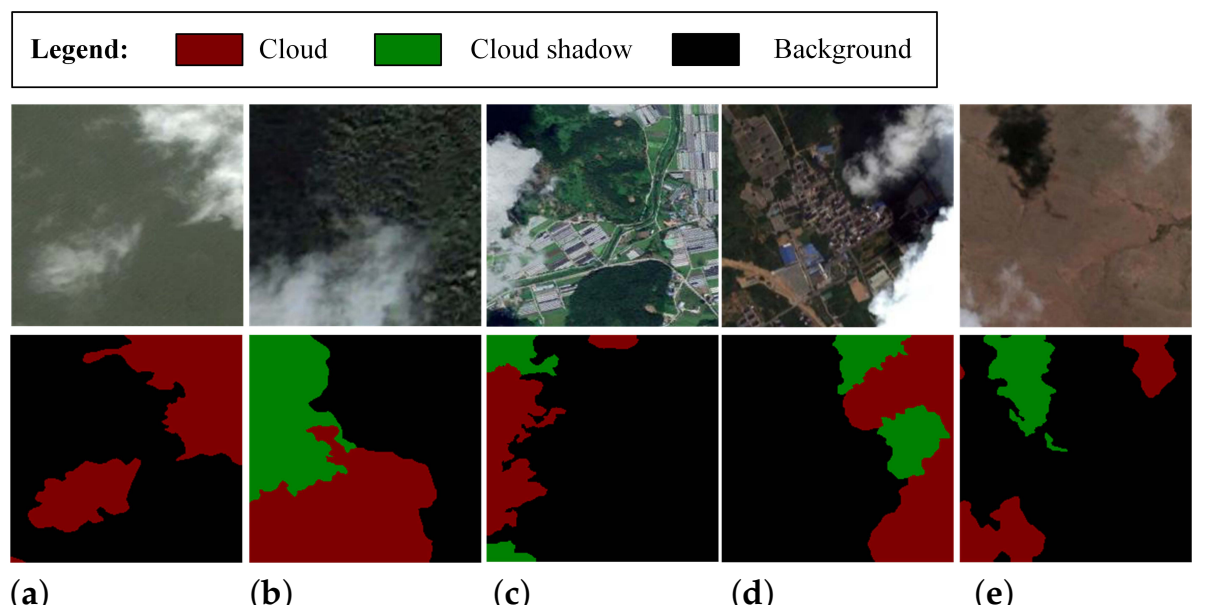

(a)

(b)

(c)

(d)

(e)

Figure 9. Display of some of the training data. (a) Water area; (b) woodland; (c) farmland; (d) cities; (e) desert.

\subsubsection{SPARCS Dataset}

The SPARCS dataset was collected from the Landsat 8 satellite, which includes two types of sensors: Operational Land Imager (OLI) and Thermal Infrared Sensor (TIRS). The band information is shown in Table 1 . The SPARCS dataset $[32,33]$ was originally created by M. Joseph Hughes of Oregon State University and manually exported from Landsat 8 OLI scenarios. Its purpose is to verify cloud and cloud shadow masking derived from the Spatial Program for Automatic Cloud and Shadow Removal (SPARCS) algorithm. The cloud validation dataset contains 80 subsets of $1000 \times 1000$ px scenarios, including cloud, cloud shadow, snow/ice, waters, and background. Limited by the video memory capacity as well, we cut the original dataset into $256 \times 256 \mathrm{px}$ and obtained a total of 1280 images. We divided the dataset into a training set and a verification set at a ratio of $8 / 2$. Due to the small size the original datasets, overfitting was prone to occur. In order to enhance the generalization ability of the model, we expanded the datasets by horizontal flipping (50\%), vertical flipping $(50 \%)$, and random rotation $\left(-30^{\circ} \sim 30^{\circ}, 50 \%\right)$ and finally obtained 2534 training sets and 634 verification sets.

Table 1. Spectral bands for the Landsat 8 sensors.

\begin{tabular}{ccc}
\hline & Landsat 8 & \\
\hline Band & Wavelength $(\mathbf{n m})$ & Resolution $(\mathbf{m})$ \\
\hline 1 (Coastal) & $430-450$ & 30 \\
2 (Blue) & $450-515$ & 30 \\
3 (Green) & $525-600$ & 30 \\
4 (Red) & $630-680$ & 30 \\
5 (NIR) & $845-885$ & 30 \\
6 (SWIR-1) & $1560-1660$ & 30 \\
7 (SWIR-2) & $2100-2300$ & 30 \\
8 (PAN) & $503-676$ & 15 \\
\hline
\end{tabular}

\subsection{Training Detail}

Our experimental implementation was based on the public platform PyTorch (Version: 0.40) (Paszke et al. 2017) [34]. In this work, we used the "Steplr" learning rate strategy, that is $l r_{\text {new }}=l r_{\text {initial }} \times \gamma^{\frac{\text { epoch }}{\text { stepsize }}}$, setting the baseline learning rate as 0.001 , the adjustment multiple as 0.95 , and the adjustment interval as 3 . A total of 200 iterations were carried out, and the cross-entropy was used as the maximum loss function. Because the Adam optimizer [35] converges quickly and stably, in the experiments involved in this article, we use Adam as the optimizer, where $\beta_{1}$ was set to 0.9 and $\beta_{2}$ to 0.999 . Our training device uses the NVIDIA GeForce RTX 3080 with a video memory capacity of $10 \mathrm{G}$, which 
is limited by the physical memory of a single GPU. When training the cloud and cloud shadow datasets, we set the batch size to 16, and when training the SPARCS dataset, we set the batch size to 6 .

In addition, we also selected the parameters of the backbone network ResNet before the comparison experiment and ablation experiment. Three groups of comparison experiments were conducted on the cloud and cloud shadow dataset, namely Resnet34, Resnet50, and Resnet101. Through the experiments, we found that the model using Resnet50 as the backbone network had the highest MIoU, indicating that Resnet50 with a moderate number of parameters was more suitable for subsequent experiments in the case of a small number of categories. See Table 2 for the relevant experiments.

Table 2. Selection of the backbone network.

\begin{tabular}{ccccc}
\hline Method & PA (\%) & MIoU (\%) & Parameter (M) & Flops (G) \\
\hline ResNet34 & 96.49 & 91.83 & 21.99 & 3.82 \\
ResNet50 & 96.68 & 92.28 & 34.49 & 5.63 \\
ResNet101 & 96.50 & 91.85 & 53.48 & 9.34 \\
\hline
\end{tabular}

\subsection{Loss Function}

Inspired by Hengshuang Zhao et al.'s setting of auxiliary loss in the pyramid scene parsing network [36], this paper also set the auxiliary loss and main loss to supervise the learning. When a network is deep, the introduction of auxiliary loss helps to optimize the learning process without affecting the learning of the main branch. In order to facilitate parameter adjustment, we introduced a total of four layers of semantic headers (see Figure 1). In the experiment, in order to ensure the fairness of the experiment, including the main loss, the weight coefficients of the two datasets were set as 1:1:1:1. In practical applications, the auxiliary loss of this model can be adjusted according to the actual situation. In addition, in order to avoid overfitting, we introduced a regularization term to the loss function. Therefore, the final loss function is defined as:

$$
L(\Theta)=\lambda_{1} L_{p}(\Theta)+\sum_{i=2}^{4} \lambda_{i} L_{a_{i}}(\Theta)+\frac{\lambda_{5}}{2}\|\Theta\|^{2},
$$

where $L_{p}$ is the principal loss, $L_{a}$ is the auxiliary loss, the $\lambda_{1}-\lambda_{5}$ ratio is set to 1 , and the network parameter $\Theta$ is updated through iterations to minimize the final loss $L(\Theta)$. The experimental results are shown in Table 3. After the auxiliary loss was added, its parameters increase slightly, but the PA (\%) and MIoU (\%) were $0.13 \%$ and $0.34 \%$ higher than the benchmark network.

Table 3. Setting the booster with auxiliary loss.

\begin{tabular}{ccccc}
\hline Method & Booster & PA (\%) & MIoU (\%) & Parameter (M) \\
\hline ResNet50 & $\times$ & 96.55 & 91.94 & 33.97 \\
ResNet50 & $\checkmark$ & 96.68 & 92.28 & 34.49 \\
\hline
\end{tabular}

\subsection{Ablation Study}

The fuse layer was composed of four components: HFE, MSC, SPSA, and SCAB. Table 4 compares the improvement of the network prediction accuracy of each module and gives the parameters and calculation amount of the model. We performed an ablation study using the cloud and cloud shadow datasets:

- Ablation for the HFE: The existing network can easily lose high-frequency information during the coding stage because of the sampling operation. The high-frequency feature extractor can recover some high-frequency components in the feature map. These high-frequency components include the texture characteristics of the cloud, which have a positive effect on the prediction of the results. As shown in Table 4, the HFE 
can increase the MIoU of the model from $92.28 \%$ to $92.56 \%$ and the PA from $96.68 \%$ to $96.81 \%$ due to the reappearance of details;

- Ablation for MSC: After high-frequency feature enhancement, the feature maps obtain more information, and multiscale convolution can extract effective multiscale information from them. As can be seen from the results in Table 4, the MSC module improved the MIoU of the model by $0.2 \%$ and the PA by $0.1 \%$;

- Ablation for SPSA: The space prior self-control module can establish the position relationship between pixels, so that classification information can greatly improve the prediction accuracy. However, at the same time, the parameter and calculation amount brought by the module are huge. MobileNet determines the influence of network width on performance [37]. The study showed that there is redundancy in the feature graph channel, which can reduce the redundancy through the compression channel, and the number of overcompressed channels will also make the information that the network can capture limited. In order to filter the redundant channel information better and reduce the parameter quantity, we suggest that the channel reduction ratio $\mathrm{R}$ be greater than or equal to 2 . As shown in Table 5, we found that when $r=4$, the performance was the best, and the parameters and the number of calculations were relatively appropriate. In addition, we added the ablation experiment of the $P_{w}$ and $P_{h}$ priors. The experimental comparison results in Table 6 showed that the model with the $P_{w}$ and $P_{h}$ priors had the best performance, which also explained how the introduction of learnable parameters help extract the spatial information from the model;

- $\quad$ Ablation for the SCAB: The feature map extracted by the multiscale module has six groups of multiscale information of different receptive fields, but the weights of this information with respect to the prediction results are different; the SPSA module establishes the location information of the feature map pixels, but ignores the channel information. Therefore, we hoped to reduce the redundancy of multiscale information by adjusting the channel weight and spatial weight of the feature map and strengthen the spatial location information to highlight the key points. Through the experiment, the MIoU of our model improved to $93.52 \%$, and the PA improved to $97.22 \%$.

Table 4. The performance comparison of the networks using the proposed modules (bold represents the best result).

\begin{tabular}{ccccc}
\hline Method & PA (\%) & MIoU (\%) & Parameter (M) & Flops (G) \\
\hline ResNet50 & 96.68 & 92.28 & 34.49 & 5.63 \\
ResNet50 + HFE & 96.81 & 92.56 & 35.19 & 5.74 \\
ResNet50 + HFE + MSC & 96.91 & 92.76 & 39.06 & 7.54 \\
ResNet50 + HFE + MSC + SPSA & 97.14 & 93.36 & 46.55 & 8.4518 \\
ResNet50 + HFE + MSC + SPSA + SCAB & $\mathbf{9 7 . 2 2}$ & $\mathbf{9 3 . 5 2}$ & 47.59 & 8.4520 \\
\hline
\end{tabular}

Table 5. The performance comparison of SPSA with different reduction rates (bold represents the best result).

\begin{tabular}{ccccc}
\hline Reduction Rate & PA (\%) & MIoU (\%) & Parameter (M) & Flops (G) \\
\hline 2 & 97.11 & 93.27 & 47.95 & 8.61 \\
4 & $\mathbf{9 7 . 1 4}$ & $\mathbf{9 3 . 3 6}$ & 46.55 & 8.45 \\
6 & 97.09 & 93.22 & 45.85 & 8.37 \\
8 & 97.09 & 93.17 & 45.50 & 8.34 \\
\hline
\end{tabular}


Table 6. The performance comparison of the self-attention modules (- represents no self-attention modules; bold represents the best result).

\begin{tabular}{ccccc}
\hline Method & PA (\%) & MIoU (\%) & Parameter (M) & Flops (G) \\
\hline- & 97.04 & 93.08 & 43.43 & 7.99 \\
SA & 97.15 & 93.36 & 47.57 & 8.44 \\
SPSA & $\mathbf{9 7 . 2 2}$ & $\mathbf{9 3 . 5 2}$ & 47.59 & 8.45 \\
\hline
\end{tabular}

\subsection{Comparison Test of the Cloud and Cloud Shadow Datasets}

In this section, we compare the proposed method with other semantic segmentation networks. In this paper, the Pixel Accuracy (PA), Mean Pixel Accuracy (MPA), F1score, Frequency-Weighted Intersection over Union (FWIoU), and Mean Intersection over Union (MIoU) were selected as the evaluation results of the experimental results. The quantitative results of the above indicators are listed in Table 7. CDUNet had the best results among all the competitive methods. By loading the pretrained model [38] on the backbone network, our network was also ahead of other models in various indicators, which shows the effectiveness of our proposed method.

Due to the space limitations, it is not possible to show the effect drawings of all models, so the six models with higher indicators and strong representation were selected for Figure 10. Because PSPNet and CCNet use a pyramid pooling module and a crisscross attention module, respectively, they are more dominant in the global receptive field. However, the details of the above two networks, such as the boundary of the cloud layer and the point cloud fragments, can not be well predicted. This is because PSPNet and CCNet lose much detail information after repeated downsampling, so they are not dominant in the presentation of cloud detail information. It can be seen from the figure that under the background of desert, water area, and forest land, the single-path models PSPnet and CCNet can only predict the general outline of clouds and cloud shadows, while multibranch networks such as HRNet and CloudNet retain some details, so the prediction of the cloud edge is more accurate. It can be observed that in the complex background, the high-frequency feature extractor designed by us fully retained the high-frequency information lost in the downsampling process, which contains rich details. At the same time, the multiscale convolution designed by us had a filtering role and filtered out unnecessary high-frequency noise. Therefore, our model can preserve the detail information to a great extent and can also completely preserve the shape and boundary of clouds and cloud shadows, which further proves the effectiveness of the proposed method.

Table 7. Results on the cloud and cloud shadow datasets. Pretrained methods are marked with "†" (bold represents the best result).

\begin{tabular}{|c|c|c|c|c|c|}
\hline Method & PA (\%) & MPA (\%) & F1 (\%) & FWIoU (\%) & MIoU (\%) \\
\hline SegNet [13] & 94.86 & 93.96 & 90.72 & 90.26 & 88.18 \\
\hline BiSeNetv2 [39] & 95.75 & 94.95 & 92.35 & 91.88 & 90.17 \\
\hline DenseASPP [40] & 95.99 & 94.44 & 93.11 & 92.35 & 90.65 \\
\hline FCN8s [11] & 96.00 & 94.92 & 92.91 & 92.35 & 90.69 \\
\hline DeepLabv3+ [41] & 96.17 & 95.32 & 93.11 & 92.66 & 91.06 \\
\hline UNet & 96.27 & 95.39 & 93.32 & 92.83 & 91.29 \\
\hline GAFRNet [7] & 96.30 & 95.50 & 93.40 & 92.90 & 91.41 \\
\hline CloudNet [8] & 96.37 & 95.61 & 93.44 & 93.01 & 91.51 \\
\hline PSPNet & 96.53 & 95.86 & 93.79 & 93.33 & 91.88 \\
\hline CCNet & 96.58 & 95.70 & 93.94 & 93.42 & 92.02 \\
\hline HRNet [42] & 96.61 & 95.78 & 93.95 & 93.47 & 92.06 \\
\hline Ours & 97.22 & 96.60 & 95.07 & 94.61 & 93.52 \\
\hline $\mathrm{FCN}_{8 \mathrm{~s}}{ }^{+}$ & 97.02 & 96.60 & 94.52 & 94.22 & 92.99 \\
\hline $\mathrm{CCNet}^{\dagger}$ & 97.13 & 96.44 & 94.88 & 94.43 & 93.26 \\
\hline PSPNet $^{\dagger}$ & 97.17 & 96.43 & 94.96 & 94.51 & 93.34 \\
\hline Ours $^{\dagger}$ & 97.51 & 96.89 & 95.56 & 95.15 & 94.12 \\
\hline
\end{tabular}




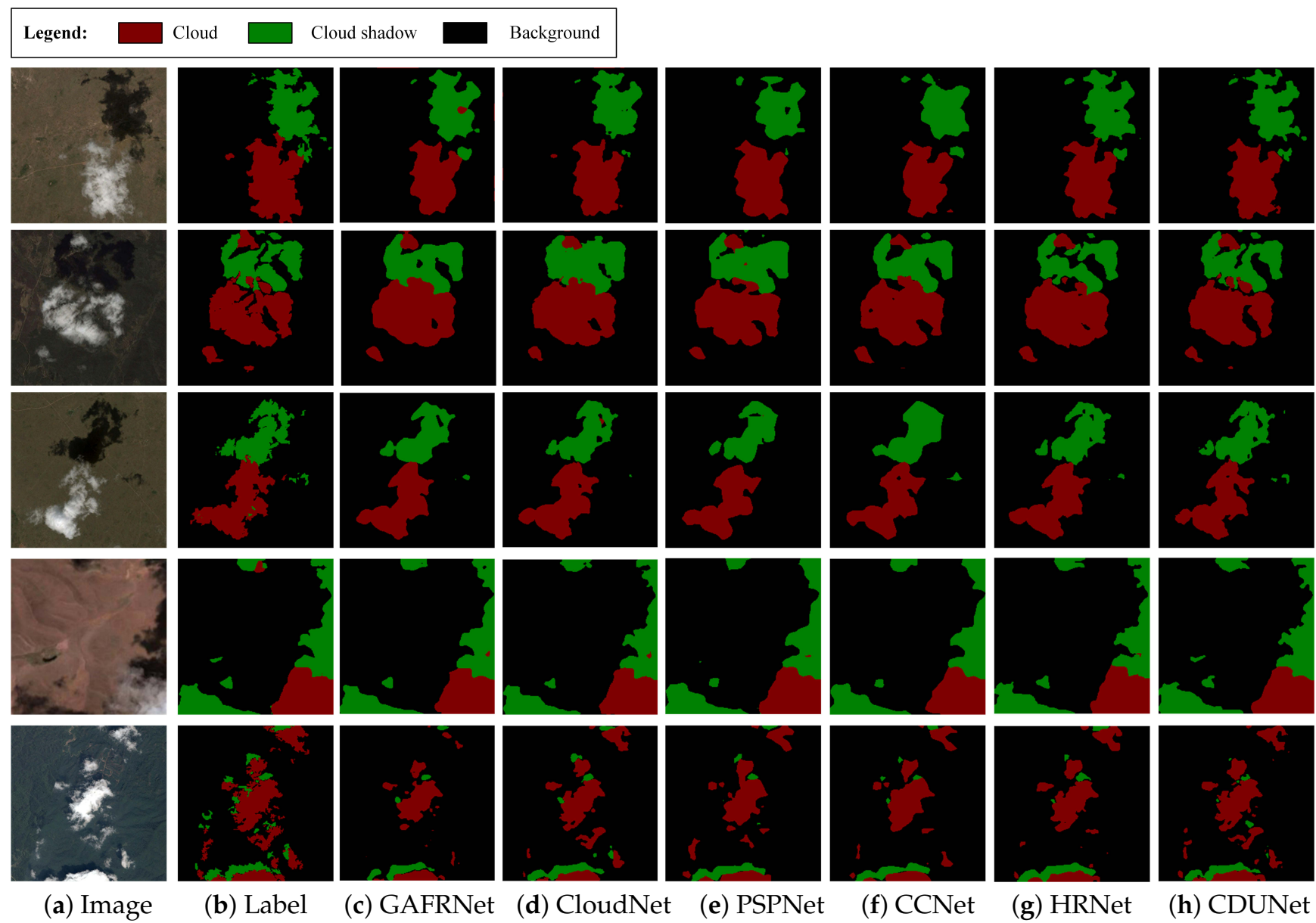

Figure 10. The prediction results of a set of test pictures. (a) The original images; (b) corresponding labels; (c) the predicted maps of GAFRNet; (d) the predicted maps of CloudNet; (e) the predicted maps of PSPNet; (f) the predicted maps of CCNet; (g) the predicted maps of HRNet; (h) the predicted maps of CDUNet.

Figure 11 shows the visual effects of different methods on cloud and cloud shadow images. The extraction of global information means that the model can reach a wider range of original images, which also means that the model can extract more global and higher semantic features. It can be seen from the figure that the segmentation edges of CloudNet and HRNet are relatively fine, but the lack of the ability of the network to extract global information leads to some category misdetection, such as the false detection of rivers and cloud shadows, thin clouds and background, and the false detection of cloud shadows and background. PSPNet adopts a pyramid structure to aggregate the global context information. This module improves the effective receptive field of the model to a great extent, but it also has an obvious shortcoming, that is it cannot establish detailed context connection. There is some relative position relationship between clouds and cloud shadows, but the global information extracted from the pyramid structure easily ignores or even destroys this correspondence, which leads to unsatisfactory prediction results. The SPSA module we designed showed better performance in dealing with this problem. Our approach was as follows: Firstly, we extracted a group of spatial vectors of a specific region and used vector multiplication to generate the spatial attention map. At the same time, we introduced a spatial prior to improve the robustness of the module. Finally, we established the context information according to the similarity between the object region features and the pixel features; the details can be reviewed in the SPSA section. The context information established by this method retains the corresponding relationship between clouds and cloud shadows as much as possible, thus effectively reducing the occurrence of false detection. It can be seen that scenes selected from a lake can also be well segmented, 
and the shape and boundary of clouds and cloud shadows can also be preserved, while other algorithms make obvious miscalculations.

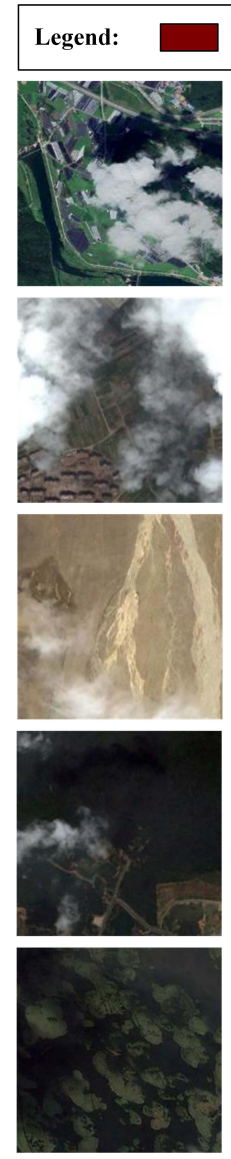

(a) Image
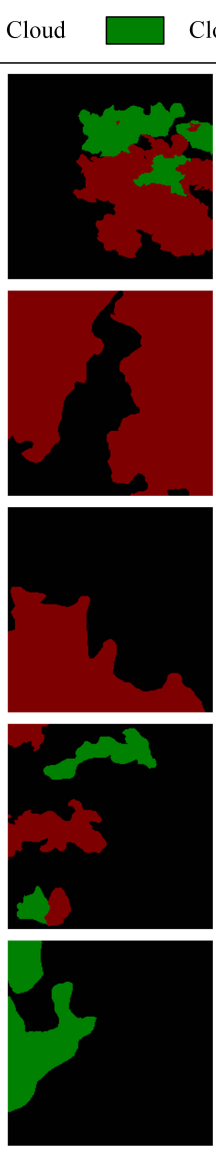

(b) Label
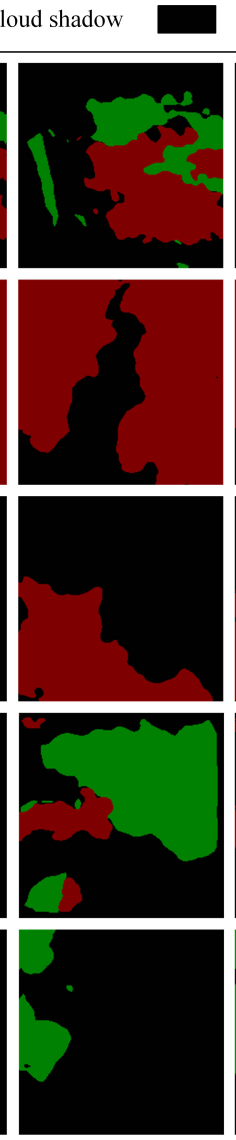

(c) GAFRNet
Background
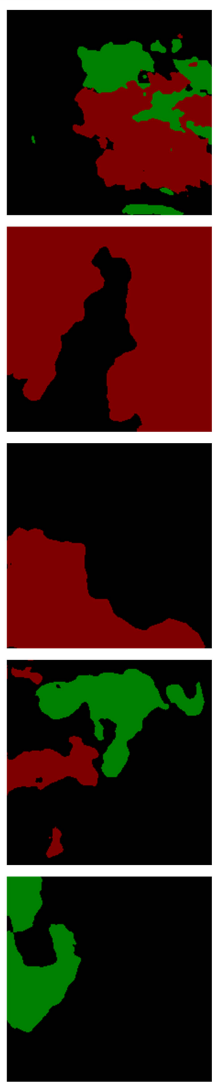

(d) CloudNet
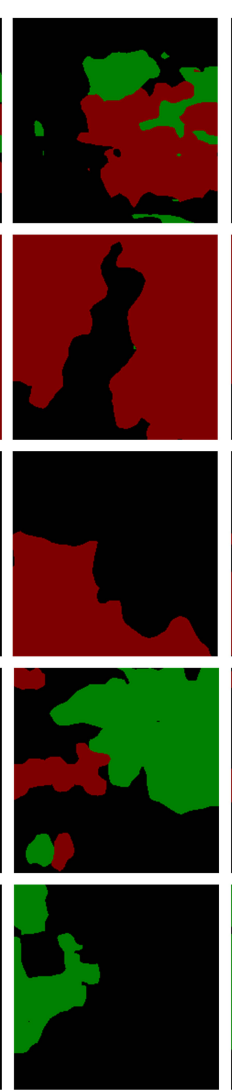

(e) PSPNet
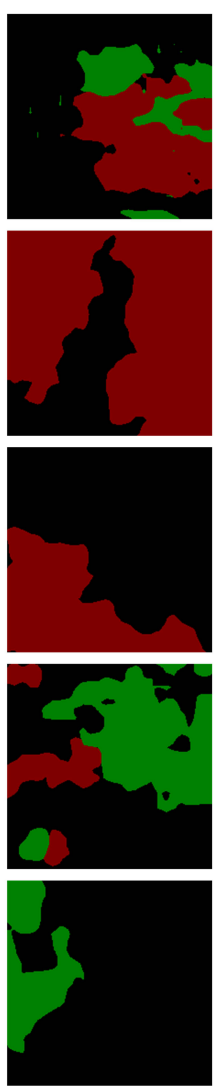

(f) $\mathrm{CCNet}$
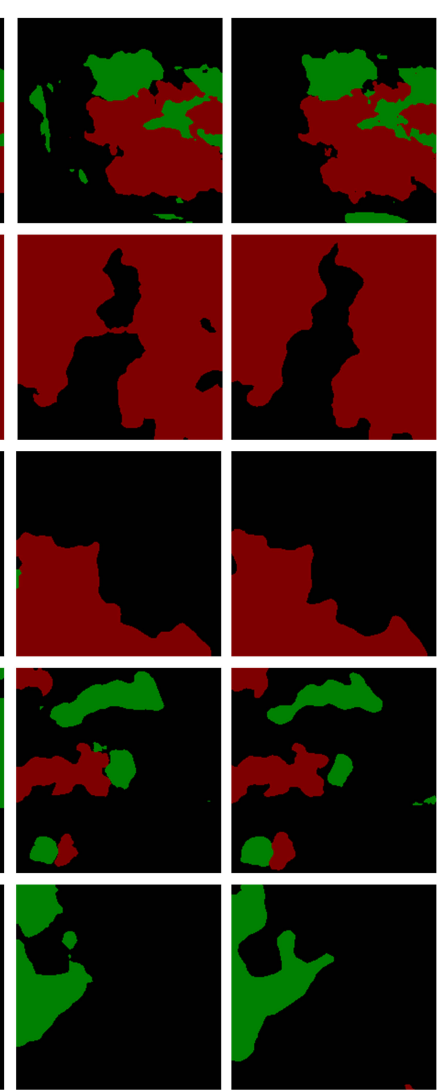

(g) HRNet

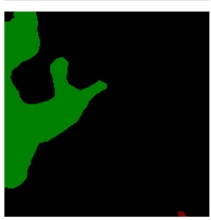

(h) CDUNet

Figure 11. The prediction results of a set of test pictures. (a) The original images; (b) corresponding labels; (c) the predicted maps of GAFRNet; (d) the predicted maps of CloudNet; (e) the predicted maps of PSPNet; (f) the predicted maps of CCNet; (g) the predicted maps of HRNet; (h) the predicted maps of CDUNet.

\subsection{Comparison Test of SPARCS Dataset}

In order to further prove the effectiveness of the proposed CDUNet, we also conducted comparative experiments on the SPARCS dataset, and the experimental results are shown in Table 8. It can be seen from the data in Table 8 that the prediction effect of UNet was better, which indicates that the U-shaped structure retained the shallow information, making it show its advantage in the more detailed label graph. Similarly, due to the support of the edge-thinning modules (HFE, MSC), our model achieved better performance in the verification set, and its indicators were significantly higher than the other networks. Table 9 shows the prediction results of each category. Through the comparison, our model had higher prediction indicators in each category than the other models, which shows that CDUNet is more accurate than other competing methods in cloud amount estimation, and it makes sense for practical remote sensing applications. 
Table 8. Results on the SPARCS testing set (bold represents the best result).

\begin{tabular}{cccccc}
\hline Method & PA (\%) & MPA (\%) & F1 (\%) & FWIoU (\%) & MIoU (\%) \\
\hline SegNet & 91.97 & 86.96 & 83.76 & 85.76 & 79.33 \\
FCN8s & 93.44 & 89.12 & 87.08 & 88.10 & 83.01 \\
DenseASPP & 93.47 & 89.20 & 87.16 & 88.18 & 83.13 \\
PSPNet & 93.53 & 89.24 & 87.16 & 88.29 & 83.15 \\
CCNet & 93.64 & 89.22 & 87.26 & 88.47 & 83.21 \\
CloudNet & 93.63 & 88.78 & 87.47 & 88.52 & 83.23 \\
DeepLabv3+ & 93.66 & 89.28 & 87.60 & 88.48 & 83.51 \\
HRNet & 93.99 & 89.75 & 87.98 & 89.07 & 84.08 \\
UNet & 94.18 & 90.27 & 88.32 & 89.37 & 84.60 \\
Ours & $\mathbf{9 5 . 2 0}$ & $\mathbf{9 2 . 2 4}$ & $\mathbf{9 0 . 4 6}$ & $\mathbf{9 1 . 0 8}$ & $\mathbf{8 7 . 3 6}$ \\
\hline
\end{tabular}

Table 9. Per class results on the SPARCS testing set (bold represents the best result).

\begin{tabular}{ccccccc}
\hline Class & Cloud (\%) & $\begin{array}{c}\text { Cloud } \\
\text { Shadow (\%) }\end{array}$ & Snow/Ice (\%) & Waters (\%) & Background (\%) & Overall (\%) \\
\hline SegNet & 82.65 & 57.67 & 89.18 & 76.78 & 90.36 & 79.33 \\
FCN8s & 84.56 & 64.85 & 90.66 & 82.74 & 92.23 & 83.01 \\
DenseASPP & 84.33 & 64.89 & 91.45 & 82.75 & 92.21 & 83.13 \\
PSPNet & 84.56 & 64.88 & 91.51 & 82.45 & 92.35 & 83.15 \\
CCNet & 85.11 & 65.47 & 91.43 & 80.93 & 92.41 & 83.23 \\
CloudNet & 84.97 & 66.17 & 91.65 & 82.69 & 92.67 & 83.51 \\
DeepLabv3+ & 84.74 & 66.58 & 91.19 & 81.92 & 92.97 & 84.60 \\
HRNet & 86.12 & 67.21 & 92.50 & 92.89 & $\mathbf{9 4 . 1 5}$ \\
UNet & 86.35 & 68.00 & 92.80 & $\mathbf{8 7 . 8 7}$ & \\
Ours & $\mathbf{8 8 . 2 9}$ & $\mathbf{7 2 . 7 4}$ & $\mathbf{9 3 . 7 3}$ & &
\end{tabular}

Figure 12 shows several examples of different types of remote sensing images. The first four images show the prediction effects of different models on thin clouds. Thin clouds are difficult to predict in cloud detection and are easily confused with ground feature information. As can be seen from the figure, because of the existence of the SPSA module, our model can distinguish different types of information better, so it can also predict thin clouds well. There are point-shaped fragmented clouds in the last four images. In our model, the advantages of the HFE and MSC modules on the edge of the clouds and the point-shaped fragmented clouds are revealed. It is represented in the figure that our model segmented the fragmented clouds more finely. At the same time, the probability of misdetection at the boundary was much less than that of the other models; even for small rivers, our model can segment well, which is not reachable for the other models. 


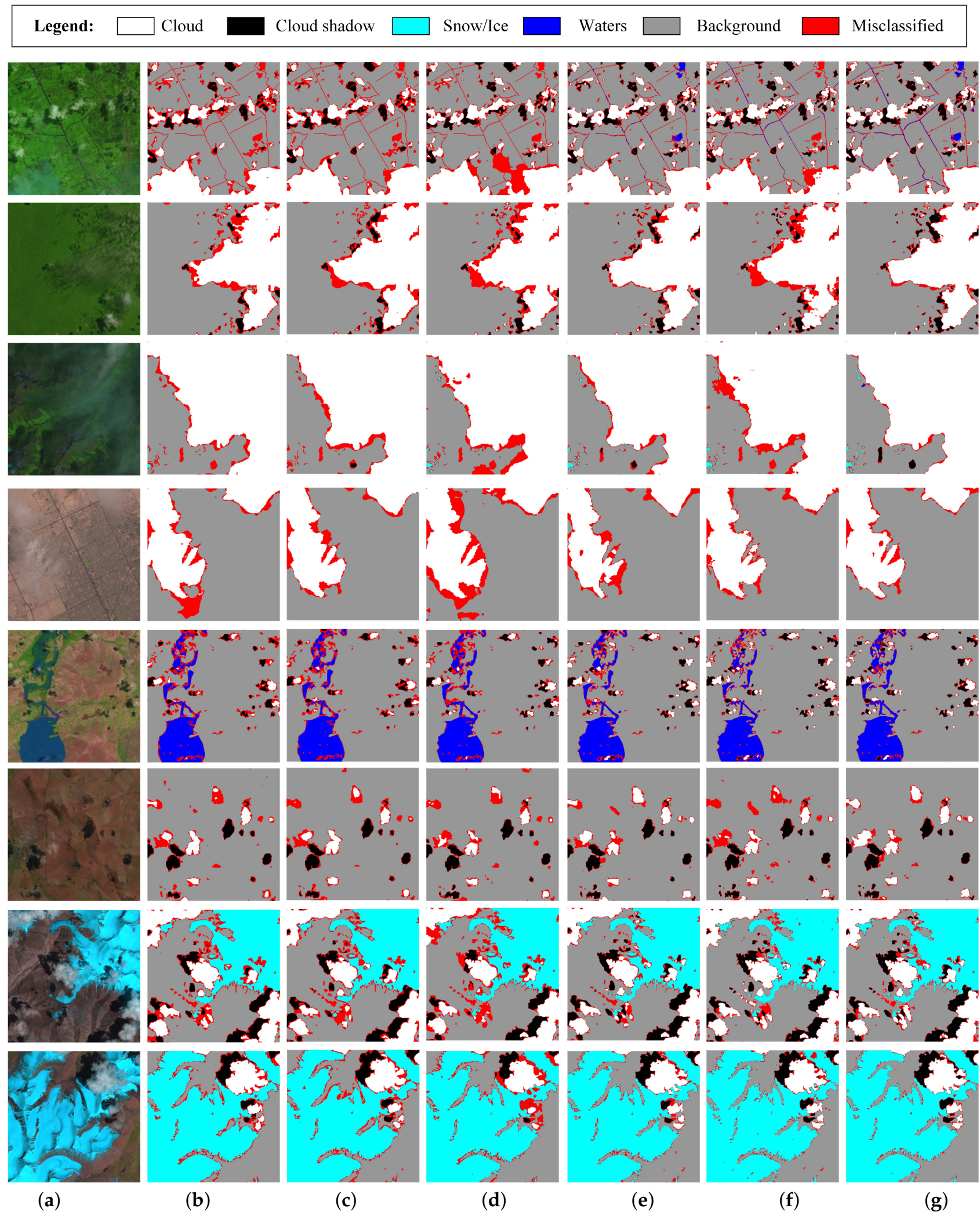

Figure 12. The prediction results of a set of test pictures. (a) The original images; (b) corresponding labels; (c) the predicted maps of UNet; (d) the predicted maps of PSPNet; (e) the predicted maps of CCNet; (f) the predicted maps of HRNet; (g) the predicted maps of proposed CDUNet. 


\section{Conclusions}

In this paper, we proposed an encoder-decoder network called CDUNet for remote sensing image cloud detection. The characteristics of the network are that it can refine the edge of cloud segmentation and can accurately locate thin clouds in the background of complex ground objects. The High-frequency Features Extractor (HFE) and the Multiscale Convolution (MSC) can compensate for the high-level semantic information diluted in the downsampling operation of the encoder, and the Spatial Prior Self-Attention (SPSA) mechanism can reconstruct the location information between categories to improve the detection accuracy. The Spatial Channel Attention Block (SCAB) can reduce the proportion of redundant information in the feature graph and improve the overall performance of the model. The experimental results showed that CDUNet achieved the most advanced cloud detection performance on the cloud and cloud shadow datasets and the SPARCS dataset. This method has a good prospect for practical applications. In the future, we will extend this method to other remote sensing images so that it can be widely used in cloud detection and cloud amount estimation. In addition, we will explore the lightweight nature of the model and further reduce the computational overhead.

Author Contributions: Conceptualization, K.H. and M.X.; methodology, M.X. and D.Z.; software, D.Z.; validation, D.Z. and K.H.; formal analysis, M.X.; investigation, M.X.; resources, M.X.; data curation, M.X. and K.H.; writing—original draft preparation, K.H.; writing—review and editing, M.X. and D.Z.; visualization, D.Z.; supervision, M.X.; project administration, M.X.; funding acquisition, M.X. All authors have read and agreed to the published version of the manuscript.

Funding: This research was funded by the National Natural Science Foundation of PR China with Grant Number 4207513.

Institutional Review Board Statement: Not applicable.

Informed Consent Statement: Not applicable.

Data Availability Statement: The data and the code of this study are available from the corresponding author upon request (xiamin@nuist.edu.cn).

Conflicts of Interest: The authors declare no conflict of interest.

\section{References}

1. Kegelmeyer, W.P., Jr. Extraction of Cloud Statistics from Whole Sky Imaging Cameras (No. SAND-94-8222); Sandia National Lab. (SNL-CA): Livermore, CA, USA, 1994.

2. Zhu, Z.; Woodcock, C.E. Object-based cloud and cloud shadow detection in Landsat imagery. Remote Sens. Environ. 2012, 118, 83-94. [CrossRef]

3. Candra, D.S.; Phinn, S.; Scarth, P. Automated cloud and cloud shadow masking for landsat 8 using multitemporal images in a variety of environments. Remote Sens. 2019,11, 2060. [CrossRef]

4. Cheng, G.; Wang, Y.; Xu, S.; Wang, H.; Xiang, S.; Pan, C. Automatic Road Detection and Centerline Extraction via Cascaded End-to-End Convolutional Neural Network. IEEE Trans. Geosci. Remote Sens. 2017, 55, 3322-3337. [CrossRef]

5. Song, L.; Xia, M.; Jin, J.; Qian, M.; Zhang, Y. SUACDNet: Attentional change detection network based on siamese U-shaped structure. Int. J. Appl. Earth Obs. Geoinf. 2021, 105, 102597. [CrossRef]

6. Wang, W.; Shi, Z. An All-Scale Feature Fusion Network With Boundary Point Prediction for Cloud Detection. IEEE Geosci. Remote Sens. Lett. 2021, 2021, 9548325. [CrossRef]

7. Xia, M.; Wang, T.; Zhang, Y.; Liu, J.; Xu, Y. Cloud/shadow segmentation based on global attention feature fusion residual network for remote sensing imagery. Int. J. Remote Sens. 2021, 42, 2022-2045. [CrossRef]

8. Mohajerani, S.; Saeedi, P. Cloud-Net: An End-To-End Cloud Detection Algorithm for Landsat 8 Imagery. In Proceedings of the IGARSS 2019-2019 IEEE International Geoscience and Remote Sensing Symposium, Yokohama, Japan, 28 July-2 August 2019; pp. 1029-1032.

9. Long, J.; Shelhamer, E.; Darrell, T. Fully convolutional networks for semantic segmentation. In Proceedings of the IEEE Conference on Computer Vision and Pattern Recognition, Boston, MA, USA, 7-12 June 2015; pp. 3431-3440. [CrossRef]

10. Zhan, Y.; Wang, J.; Shi, J.; Cheng, G.; Yao, L.; Sun, W. Distinguishing Cloud and Snow in Satellite Images via Deep Convolutional Network. IEEE Geosci. Remote Sens. Lett. 2017, 14, 1785-1789. [CrossRef]

11. Drönner, J.; Korfhage, N.; Egli, S.; Mühling, M.; Thies, B.; Bendix, J.; Freisleben, B.; Seeger, B. Fast Cloud Segmentation Using Convolutional Neural Networks. Remote Sens. 2018, 10, 1782. [CrossRef] 
12. Chai, D.; Newsam, S.; Zhang, H.K.; Qiu, Y.; Huang, J. Cloud and cloud shadow detection in Landsat imagery based on deep convolutional neural networks. Remote Sens. Environ. 2019, 225, 307-316. [CrossRef]

13. Badrinarayanan, V.; Kendall, A.; Cipolla, R. SegNet: A Deep Convolutional Encoder-Decoder Architecture for Image Segmentation. IEEE Trans. Pattern Anal. Mach. Intell. 2017, 39, 2481-2495. [CrossRef]

14. Ronneberger, O.; Fischer, P.; Brox, T. U-Net: Convolutional Networks for Biomedical Image Segmentation. In Proceedings of the International Conference on Medical Image Computing and Computer-Assisted Intervention, Munich, Germany, 5-9 October 2015; pp. 234-241._28. [CrossRef]

15. He, K.; Zhang, X.; Ren, S.; Sun, J. Deep residual learning for image recognition. In Proceedings of the IEEE Conference on Computer Vision and Pattern Recognition, Las Vegas, NV, USA, 27-30 June 2016; pp. 770-778. [CrossRef]

16. Qu, Y.; Xia, M.; Zhang, Y. Strip pooling channel spatial attention network for the segmentation of cloud and cloud shadow. Comput. Geosci. 2021, 157, 104940. [CrossRef]

17. Xia, M.; Liu, W.; Wang, K.; Song, W.; Chen, C.; Li, Y. Non-intrusive load disaggregation based on composite deep long short-term memory network. Expert Syst. Appl. 2020, 160, 113669. [CrossRef]

18. Wang, Z.; Xia, M.; Lu, M.; Pan, L.; Liu, J. Parameter Identification in Power Transmission Systems Based on Graph Convolution Network. IEEE Trans. Power Deliv. 2021, 1. [CrossRef]

19. Xia, M.; Liu, W.; Shi, B.; Weng, L.; Liu, J. Cloud/snow recognition for multispectral satellite imagery based on a multidimensional deep residual network. Int. J. Remote Sens. 2018, 40, 156-170. [CrossRef]

20. Xia, M.; Zhang, X.; Liu, W.; Weng, L.; Xu, Z. Multi-Stage Feature Constraints Learning for Age Estimation. IEEE Trans. Inf. Forensics Secur. 2020, 15, 2417-2428. [CrossRef]

21. Lin, M.; Chen, Q. Network in network. arXiv 2013, arXiv:1312.4400.

22. LeCun, Y.; Bottou, L.; Bengio, Y.; Haffner, P. Gradient-based learning applied to document recognition. Proc. IEEE 1998, 86, 2278-2324. [CrossRef]

23. Chen, C.F.; Fan, Q.; Mallinar, N.; Sercu, T.; Feris, R. Big-little net: An efficient multiscale feature representation for visual and speech recognition. arXiv 2018, arXiv:1807.03848.

24. Chen, Y.; Fan, H.; Xu, B.; Yan, Z.; Kalantidis, Y.; Rohrbach, M.; Shuicheng, Y.; Feng, J. Drop an Octave: Reducing Spatial Redundancy in Convolutional Neural Networks With Octave Convolution. In Proceedings of the 2019 IEEE/CVF International Conference on Computer Vision (ICCV), Seoul, Korea, 27-28 October 2019; pp. 3435-3444.

25. Cheng, B.; Xiao, R.; Wang, J.; Huang, T.; Zhang, L. High frequency residual learning for multiscale image classification. arXiv 2019, arXiv:1905.02649.

26. Luo, W.; Li, Y.; Urtasun, R.; Zemel, R. Understanding the effective receptive field in deep convolutional neural networks . In Proceedings of the 30th International Conference on Neural Information Processing Systems, Barcelona, Spain, 5-10 December 2016; pp. 4905-4913.

27. Wang, X.; Girshick, R.; Gupta, A.; He, K. Non-local Neural Networks. In Proceedings of the IEEE/CVF Conference on Computer Vision and Pattern Recognition (CVPR), Salt Lake City, UT, USA, 18-22 June 2018; pp. 7794-7803. [CrossRef]

28. Fu, J.; Liu, J.; Tian, H.; Li, Y.; Bao, Y.; Fang, Z.; Lu, H. Dual Attention Network for Scene Segmentation. In Proceedings of the IEEE/CVF Conference on Computer Vision and Pattern Recognition (CVPR), Long Beach, CA, USA, 16-20 June 2019; pp. 3141-3149.

29. Huang, Z.; Wang, X.; Huang, L.; Huang, C.; Wei, Y.; Liu, W. CCNet: Criss-Cross Attention for Semantic Segmentation. In Proceedings of the 2019 IEEE/CVF International Conference on Computer Vision (ICCV), Seoul, Korea, 27 October-2 November 2019; pp. 603-612.

30. Li, X.; Wang, W.; Hu, X.; Yang, J. Selective kernel networks. In Proceedings of the 2019 IEEE/CVF Conference on Computer Vision and Pattern Recognition (CVPR), Long Beach, CA, USA, 15-20 June 2019; pp. 510-519.

31. Woo, S.; Park, J.; Lee, J.-Y. CBAM: Convolutional Block Attention Module. arXiv 2018, arXiv:1807.06521.

32. Hughes, M. L8 SPARCS Cloud Validation Masks; US Geological Survey: Sioux Falls, SD, USA, 2016.

33. Hughes, M.J.; Hayes, D.J. Automated detection of cloud and cloud shadow in single-date Landsat imagery using neural networks and spatial postprocessing. Remote Sens. 2014, 6, 4907-4926. [CrossRef]

34. Paszke, A.; Gross, S.; Chintala, S.; Chanan, G.; Yang, E.; DeVito, Z.; Lin, Z.; Desmaison, A.; Antiga, L.; Lerer, A. Automatic Differentiation in Pytorch. In Proceedings of the NIPS 2017 Workshop Autodiff Submission, Long Beach, CA, USA, 9 December 2017.

35. Kingma, D.P.; Ba, J. Adam: A method for stochastic optimization. In Proceedings of the International Conference Learn (ICLR), San Diego, CA, USA, 5-8 May 2015.

36. Zhao, H.; Shi, J.; Qi, X.; Wang, X.; Jia, J. Pyramid Scene Parsing Network. In Proceedings of the 2017 IEEE Conference on Computer Vision and Pattern Recognition (CVPR), Honolulu, HI, USA, 21-26 July 2017; 630-645.

37. Howard, A.G.; Zhu, M.; Chen, B.; Kalenichenko, D.; Wang, W.; Wey, T.; Andreetto, M.; Adam, H. Mobilenets: Efficient convolutional neural networks for mobile vision applications. arXiv 2017, arXiv:1704.04861.

38. Russakovsky, O.; Deng, J.; Su, H.; Krause, J.; Satheesh, S.; Ma, S.; Huang, Z.; Karpathy, A.; Khosla, A.; Bernstein, M.; et al. Imagenet large scale visual recognition challenge. Int. J. Comput. Vis. 2015, 115, 211-252. [CrossRef]

39. Yu, C.; Gao, C.; Wang, J.; Yu, G.; Shen, C.; Sang, N. BiSeNet V2: Bilateral Network with Guided Aggregation for Real-Time Semantic Segmentation. Int. J. Comput. Vis. 2021, 129, 3051-3068. [CrossRef] 
40. Yang, M.; Yu, K.; Zhang, C.; Li, Z.; Yang, K. DenseASPP for Semantic Segmentation in Street Scenes. In Proceedings of the 2018 IEEE/CVF Conference on Computer Vision and Pattern Recognition, Salt Lake City, UT, USA, 18-23 June 2018; pp. 3684-3692.

41. Chen, L.C.; Zhu, Y.; Papandreou, G.; Schroff, F.; Adam, H. Encoder-decoder with atrous separable convolution for semantic image segmentation. In Proceedings of the European Conference on Computer Vision (ECCV), Munich, Germany, 8-14 September 2018; pp. 801-818.

42. Sun, K.; Xiao, B.; Liu, D.; Wang, J. Deep High-Resolution Representation Learning for Human Pose Estimation. In Proceedings of the IEEE/CVF Conference on Computer Vision and Pattern Recognition (CVPR), Long Beach, CA, USA, 16-20 June 2019; pp. 5686-5696. [CrossRef] 